\title{
Assessment of changes in liver blood flow after food intake- comparison of ICG clearance and echo-Doppler
}

\author{
J. BURGGRAAF, H. C. SCHOEMAKER \& A. F. COHEN \\ Centre for Human Drug Research, Zernikedreef 10, Leiden, The Netherlands
}

Echo-Doppler measurements of portal venous blood flow in intrahepatic branches and indocyanine green (ICG) clearance after continuous i.v. infusion as measure for liver blood flow were compared to evaluate the increase in splanchnic blood flow after food intake. It was shown that both methods assessed the changes in flow in a similar manner. Changes in blood flow in intrahepatic portal vein branches measured with echo-Doppler adequately predicted the change in ICG concentrations. Hence, echo-Doppler measurements of hepatic portal blood flow in intrahepatic branches can be used to estimate changes in total liver blood flow.

\section{Keywords liver blood flow ICG clearance echo-Doppler}

\section{Introduction}

Echo-Doppler measurements of hepatic portal venous blood flow are widely applied in qualitative diagnostic procedures. The technique is also used to quantify total portal blood flow, but the measured volumes are generally smaller than expected from physiology [1-4], indicating that assessment is less precise than desired. This has resulted in the application of the ultrasound technique to evaluate changes in flow rather than absolute flow volumes. In any case, assessment of flow volume in the main portal vein remains difficult. Problems such as the estimation of the cross-sectional area of the vessel and unreliable velocity estimates due to large angles of insonation are essentially unsolved when applying the technique to the main portal vein $[5,6]$. Most of the difficulties can be circumvented by evaluating changes in portal blood flow measured in smaller intra-hepatic branches as shown by previous experiments $[7,8]$. With this approach all requirements for accurate measurements are met. However, the validity of this technique is unknown. Therefore, the influence of food intake on liver blood flow was evaluated by simultaneous measurement of echoDoppler flow of a portal branch, and the standard indocyanine green (ICG) clearance technique. This study was part of another study already published [9].

\section{Methods}

Ten young, healthy, male volunteers participated, after written informed consent, in the study which was approved by the ethics committee of Leiden University Hospital. The subjects were studied after an overnight fast. After i.v. cannulation, the subjects were placed in a sitting position which was maintained for the entire experiment. After $1 \mathrm{~h}$ rest, ICG (Cardio-Green ${ }^{\circledR}$, Becton Dickinson and Co., Cockeysville, MD, USA) was infused continuously for $150 \mathrm{~min}$ (total dose: $90 \mathrm{mg}$ ). One hour after the start of the infusion, the subjects consumed a standardized meal (caloric content: $2520 \mathrm{~kJ} ; 25 \%$ energy protein, 51\% energy lipid, 24\% energy carbohydrate). Blood samples for ICG assay were drawn regularly. ICG concentrations were measured using h.p.l.c. [10]. Flow measurements of a portal vein branch were made using a Toshiba SSA-250A echograph with pulsed Doppler before (twice) and at 15, 30, 45, 60, 80, 100 and $120 \mathrm{~min}$ after food intake, as described previously $[7,9]$.

\section{Data analysis}

For each subject the average ICG plasma concentration of the three time points before food intake $(53,56$ and 59 min after the start of the ICG infusion) was calculated. The group average of these pre-food ICG concentrations (Cavg) was used to calculate the total ICG blood clearance:

$$
\mathrm{CL}=\mathrm{R}_{\mathrm{O}} /\left(\mathrm{Cavg}^{*}(1-\mathrm{Ht})\right)
$$

where $R_{O}$ is the ICG infusion rate and $\mathrm{Ht}$ is the haematocrit. Assuming that the ICG extraction ratio (E) for this study population was 0.72 (mean value calculated from 10 studies reported in the literature), 

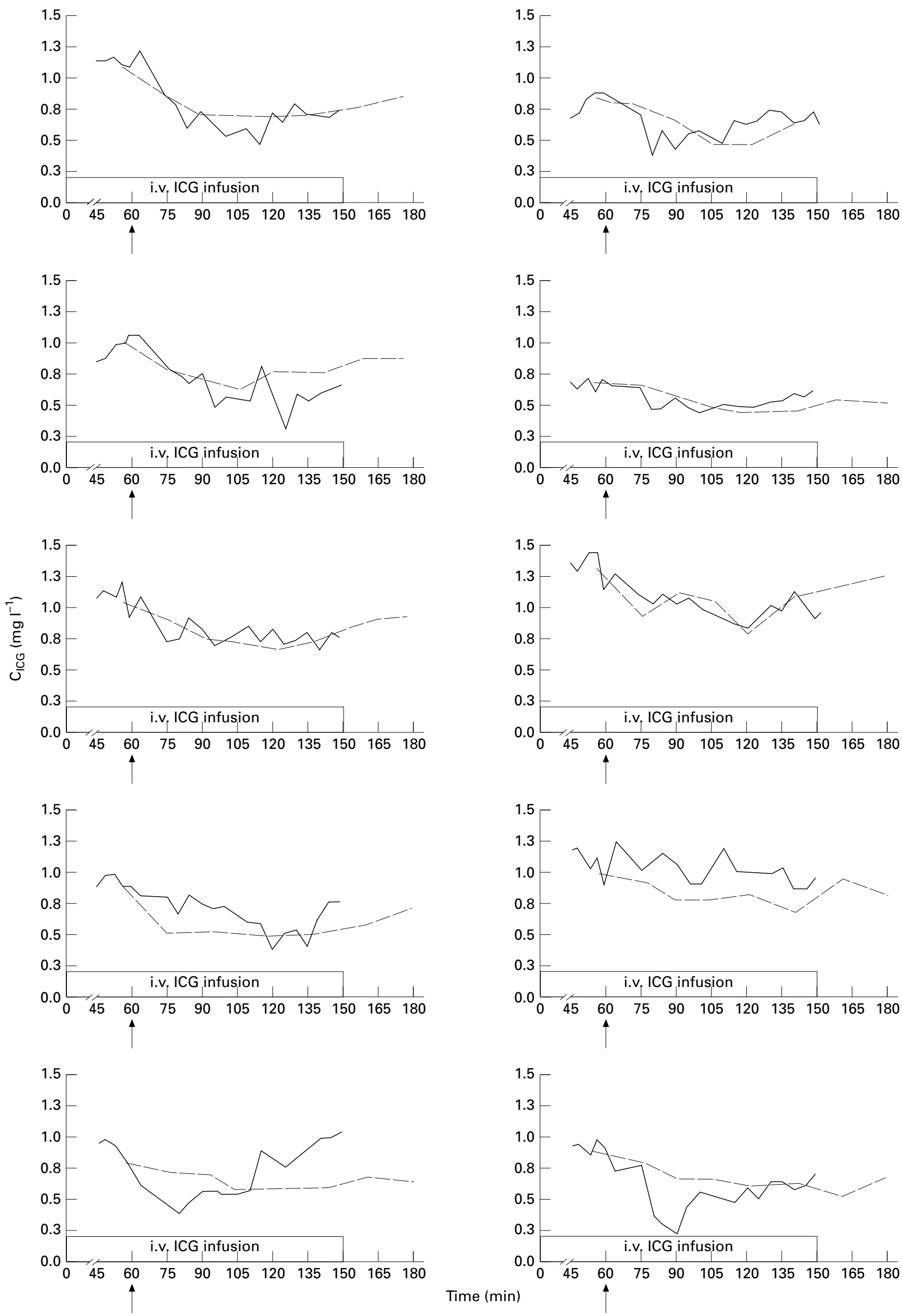

Figure 1 Individual ICG concentrations-time profiles as measured (solid lines) and predicted from echo-Doppler measurements (broken lines). ICG infusion is indicated with the box and start of food intake is marked by arrow. 
the average basal liver blood flow $(\mathrm{Q})$

$$
\mathrm{Q}=\mathrm{CL} / \mathrm{E}
$$

and the average intrinsic clearance

$$
\mathrm{E}=\mathrm{CL}_{\text {int }} /\left(\mathrm{CL}_{\mathrm{int}}+\mathrm{Q}\right)
$$

were calculated. The average intrinsic clearance (3.91 $\mathrm{min}^{-1}$ ) was subsequently used to calculate each individual's basal liver blood flow $\left(\mathrm{Q}_{\text {basal }}\right)$ by using the following equation

$$
\text { Cpre }=\left(\mathrm{R}_{\mathrm{O}} * \mathrm{Q}_{\text {basal }} * \mathrm{CL}_{\text {int }}\right) /\left(\left(\mathrm{CL}_{\text {int }}+\mathrm{Q}_{\text {basal }}\right) *(1-\mathrm{Ht})\right)
$$

For each subject, portal blood flow volumes measured at the time points after food intake were expressed as percentage change from the average flow value before food intake. These percentage changes were used to calculate total liver blood flow at the different time points $\left(\mathrm{Q}_{t}\right)$. Assuming that changes in liver blood flow are of sufficient duration, equation 4 can be re-formulated to calculate ICG concentrations at different time points after food intake:

$$
\mathrm{C}_{\mathrm{ICG}, t}=\left(\mathrm{R}_{\mathrm{O}} * \mathrm{Q}_{t}{ }^{*} \mathrm{CL}_{\text {int }}\right) /\left(\left(\mathrm{CL}_{\mathrm{int}}+\mathrm{Q}_{t}\right) *(1-\mathrm{Ht})\right)
$$

in which subscript ' $t$ ' denotes time.

\section{Results}

Basal liver blood flow was 1.6 (s.d.: 0.5) $1 \mathrm{~min}^{-1}$ (range: 1.0-2.61 $\mathrm{min}^{-1}$ ). The individual time courses of the measured ICG plasma concentrations and the ICG concentrations predicted from the echo-Doppler results are shown in Figure 1. Both assessments of liver blood flow agreed well in most subjects. Although deviations between predicted and measured curves were present in some individuals, they appear not to be systematic. This is further illustrated by Figure 2 which shows the average curves.

\section{Discussion}

Liver blood flow is commonly assessed through measurement of the ICG clearance following bolus injection or continuous infusion [11]. Although clearance techniques have an established role, there are some disadvantages. They are invasive, requiring access to the circulation for administration and blood sampling. The methods ideally require determination of the extraction ratio by catheterization of the hepatic vein, which is seldom justified in healthy volunteers. The use of the method is limited by the maximal tolerated dose to a few times within a single experiment. The timing of repeated assessments with consecutive bolus injections may be difficult. Finally, the method is relatively insensitive to increases in flow [7].

Echo-Doppler measurements of blood flow in intrahepatic branches can be used to estimate changes

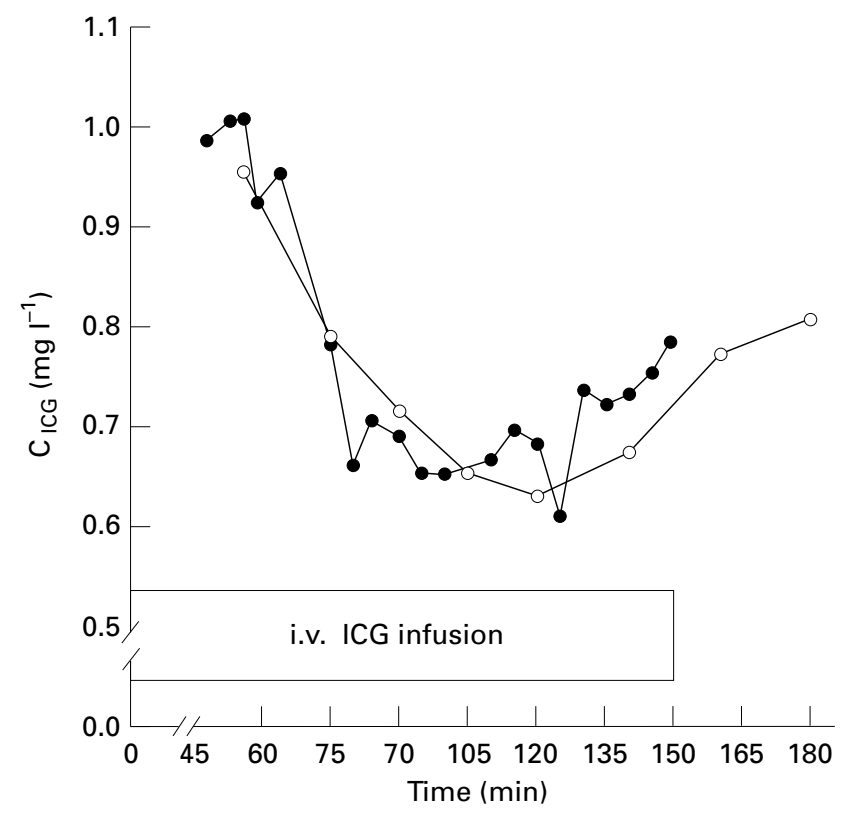

Figure 2. Average ICG $(n=10)$ concentration-time profile observed (closed symbols) during continuous ICG infusion and food intake at $60 \mathrm{~min}$. The open symbols show the ICG concentrations predicted to occur using echo-Doppler portal blood flow measurements.

in total liver blood flow, as shown by the agreement between the predicted and observed ICG concentrations. By showing the individual curves an impression of the correspondence between both methods is given. This was preferred over reporting for instance correlation coefficients between measured and predicted values because this measure heavily relies on the spread of the data and is often misused as a measure for goodness of fit. It thus appears that the measured ICG concentrations were well predicted by the Doppler measurements. Although deviations between predicted and measured curves were present in some individuals, they were not systematic.

It is important to point out that a few assumptions underlie these findings. Firstly, a basal extraction ratio for ICG had to be assumed from the literature. Secondly, changes in portal blood flow were assumed to be representative for changes in total liver blood flow. Although portal venous and hepatic artery flow can be reciprocally linked, this is certainly not always the case as shown by the effects of many gastrointestinal hormones released during food intake [12]. Thirdly, equation 5 used to calculate the ICG concentrations after food intake is based upon steady state conditions. In this study with an elevated liver blood flow after food intake, and consequently a short half-life of ICG (2-3 min) rapid attainment of new steady state conditions within the echo-Doppler measurement could be expected. Finally, this experiment lacked a 'control' treatment arm. However, previous experiments have shown that both ICG concentrations and portal blood flow are very stable over the time span $(2-3 \mathrm{~h})$ of this study [7].

This study demonstrated that repetitive echo-Doppler measurements of intra-hepatic portal vein branches can be used to evaluate changes in portal blood flow after 
food intake. Furthermore, changes in regional portal blood flow can be extrapolated to total hepatic flow, as shown by the agreement between observed and predicted ICG concentrations.

\section{References}

1 de Vries PJ, van Hattum J, Hoekstra JBL, de Hooge P. Duplex Doppler measurement of portal venous flow in normal subjects: inter- and intra-observer variability. J Hepatol 1991; 13: 358-363.

2 Cooper AM, Braatvedt GD, Qamar M, et al. Fasting and post-prandial splanchnic blood flow is reduced by a somatostatin analogue (octreotide) in man. Clin Sci 1991; 81: $169-175$.

3 Carlisle KM, Halliwell M, Read AE, Wells PNT. Estimation of total hepatic blood flow by duplex ultrasound. Gut 1992; 33: 92-97.

4 Ray-Chaudhuri K, Thomaides T, Maule S, Watson L, Lowe S, Mathias CJ. The effect of captopril on the superior mesenteric artery and portal venous blood flow in normal man. Br J Clin Pharmacol 1993; 35: 517-524.

5 Gill RW. Measurement of blood flow by ultrasound: accuracy and sources of error. Ultrasound Med Biol 1985; 11: $625-641$.

6 Taylor KJW, Holland S. Doppler US PartI. Basic principles, instrumentation and pitfalls. Radiology 1990; 174: $297-307$.

7 Burggraaf J, Schoemaker HC, Kroon JM, Huisman LGM, Kluft C, Cohen AF. The influence of dDAVP on endogenous fibrinolysis, haemodynamics and liver blood flow in healthy subjects. Clin Sci 1994; 86: 497-503.

8 Horn JR, Zierler B, Bauer LA, Reiss W, Strandness Jr DE. Estimation of hepatic blood flow in branches of hepatic vessels utilizing a noninvasive, duplex Doppler method. J Clin Pharmacol 1990; 30: 922-929.

9 Griensven van JMT, Burggraaf J, Gerloff J, et al. Effects of changing liver blood flow by exercise and food on kinetics and dynamics of saruplase. Clin Pharmacol Ther 1995; 57: 381-389.

10 Rappaport PL, Thiessen JJ. High-pressure liquid chromatographic analysis of indocyanine green. J Pharm Sci 1982; 71: $157-161$.

11 Soons PA, DeBoer A, Cohen AF, Breimer DD. Assessment of hepatic blood flow in healthy subjects by continuous infusion of indocyanine green. Br J Clin Pharmacol 1991; 32: 697-704.

12 Kawasaki T, Carmichael FJ, Saldivia V, Roldan L, Orrego H. Relationship between portal venous and hepatic arterial blood flows: spectrum of response. Am J Physiol 1990; 259: G1010-G1018.

(Received 2 January 1996, accepted 8 May 1996) 